\title{
Sistemas de expresión de proteínas recombinantes para el análisis funcional de antígenos de Plasmodium falciparum y Plasmodium vivax: una revisión
}

\author{
Álida Marcela Gómez Rodríguez¹ (D), Laura Esperanza Cuy Chaparro² (D), Anny Jineth Camargo \\ Mancipe $^{1}$ (D)
}

\section{RESUMEN}

Introducción: Para diseñar vacunas es necesario comprender la función de los antígenos de Plasmodium spp. involucrados en la invasión a células hospederas. Diferentes investigaciones han generado proteínas recombinantes utilizando sistemas de expresión heterólogos y así han obtenido moléculas semejantes a las nativas. Con estos avances se desarrollan estrategias que bloquean la infección de estos patógenos.

Objetivo: Describir las características y los aspectos metodológicos más importantes de los sistemas de expresión de las proteínas recombinantes en estudios funcionales de Plasmodium spp.

Metodología: Revisión descriptiva de estudios publicados en Pubmed, Science Direct, Embase y Medline, entre 2010 y 2020, que incluyeran sistemas recombinantes en células de Escherichia coli, de mamífero y sistemas libres de células, para estudios funcionales de antígenos de Plasmodium falciparum y Plasmodium vivax. Se revisaron 70 artículos originales y 58 cumplieron con los criterios establecidos.

Resultados: Obtener proteínas recombinantes mediante un sistema procariota, de mayor rendimiento y bajo costo, ha permitido estudiar un número importante de antígenos. Los sistemas con células de mamífero y libres de células, que permiten modificaciones postraduccionales y plegamiento adecuado de moléculas, se usan para producir librerías de antígenos con estructura conformacional similar a la nativa.

Conclusión: El estudio de los antígenos de Plasmodium spp. implicados en la infección y desarrollo de células diana requiere una adecuada selección del método de producción recombinante. El refinamiento de procesos de expresión en sistemas procariotas, eucariotas e in vitro, mediante ingeniería genética y cultivo celular, permitirá mejores rendimientos y menor costo.

Palabras clave: antígenos; expresión génica; malaria; Plasmodium falciparum; Plasmodium vivax.

${ }^{1}$ Universidad del Rosario, Bogotá, Colombia. Universidad de Boyacá, Tunja, Colombia .

2 Universidad del Rosario, Bogotá, Colombia.

Autora de correspondencia: Álida Marcela Gómez Rodríguez. Correo electrónico: aligomez@uniboyaca.edu.co.

Citar este artículo así:

Gómez Rodríguez AM, Cuy Chaparro LE, Camargo Mancipe AJ. Sistemas de expresión de proteínas recombinantes para el análisis funcional de antígenos de Plasmodium falciparum y Plasmodium vivax: una revisión. Rev Investig Salud Univ Boyacá. 2021;8(2): 110-130. https://doi. org/10.24267/23897325.484 


\title{
Recombinant Protein Expression Systems for Functional Analysis of Plasmodium falciparum and Plasmodium vivax Antigens: A Review
}

\begin{abstract}
Introduction: Understanding the function of Plasmodium spp. Antigens involved in invasion of host cells is necessary to design vaccines. Different studies have generated recombinant proteins using heterologous expression systems, obtaining molecules similar to native ones. These advances are essential to develop strategies that block the infection of these pathogens.
\end{abstract}

Objective: Describe the most important characteristics and methodological aspects of recombinant protein expression systems in functional studies of Plasmodium spp.

Methodology: Descriptive review of studies published in Pubmed, Science Direct, Embase and Medline, between 2010 and 2020, that included recombinant systems in Escherichia coli cells, mammalian and cell-free, for functional studies of Plasmodium falciparum and Plasmodium vivax antigens. 70 original articles were reviewed, 58 met the established criteria.

Results: Obtaining recombinant proteins by means of a prokaryotic system, with higher performance and low cost, has allowed functional studies of a significant number of antigens. Mammalian cell and cell free systems, which allow for post-translational modifications and adequate folding of molecules, are used to produce antigen libraries with native-like conformational structure.

Conclusion: Plasmodium spp. antigen study involved in infection and development in target cells, requires adequate selection of the recombinant production method. The refinement of expression processes in prokaryotic, eukaryotic and in vitro systems, through genetic engineering and cell culture, will allow better yields and lower cost

Keywords: antigens; malaria; gene expression; Plasmodium falciparum; Plasmodium vivax. 


\title{
Sistemas de expressão de proteínas recombinantes para o analise funcional de antígenos de Plasmodium falciparum e Plasmodium vivax: uma revisão
}

\begin{abstract}
RESUMO
Introdução: Para desenvolver vacinas, é necessário entender a função dos antígenos de Plasmodium spp. envolvidos na invasão das células hospedeiras. As pesquisas têm gerado proteínas recombinantes utilizando sistemas de expressão heterólogos para obter moléculas similares às nativas. Com estes avanços, estratégias que bloqueiam a infecção destes patógenos estão sendo desenvolvidas.

Objetivo: Descrever as características mais importantes e aspectos metodológicos dos sistemas de expressão de proteínas recombinantes em estudos funcionais de Plasmodium spp.

Metodologia: Revisão descritiva dos estudos publicados em Pubmed, Science Direct, Embase e Medline, entre 2010 e 2020, que incluíram sistemas recombinantes em células de Escherichia coli, de mamífero e sistemas livres de células, para estudos funcionais dos antígenos de Plasmodium falciparum e Plasmodium vivax. Setenta artigos originais foram revisados e 58 preenchiam os critérios estabelecidos.
\end{abstract}

Resultado: A obtenção de proteínas recombinantes usando um sistema procariótico, com maior rendimento e baixo custo, permitiu o estudo de um número significativo de antígenos. Sistemas de células mamíferas e sem células, que permitem modificações pós-tradução e dobramento adequado das moléculas, são usados para produzir bibliotecas de antígenos com uma estrutura semelhante à nativa.

Conclusão: O estudo dos antígenos Plasmodium spp. envolvidos na infecção e no desenvolvimento das células-alvo requer uma seleção adequada do método de produção recombinante. $O$ refinamento dos processos de expressão em sistemas procarióticos, eucarióticos e in vitro, através da engenharia genética e da cultura celular, permitirá melhores rendimentos e menores custos.

Palavras-chave: antígenos; expressão gênica; malária; Plasmodium falciparum; Plasmodium vivax. 


\section{INTRODUCCIÓN}

La malaria se considera un importante problema de salud pública en el mundo, al punto que es una de las principales causas de mortalidad y morbilidad humana en muchos países tropicales y subtropicales (1). Esta enfermedad la ocasionan parásitos del género Plasmodium, que son transmitidos al hombre por la picadura de mosquitos hembra infectados del género Anopheles (2).

La mayoría de los estudios e investigaciones se han enfocado en el descubrimiento de una vacuna contra Plasmodium falciparum, por ser la especie más ampliamente distribuida en el continente africano y que causa el tipo de malaria más letal y severa en los humanos. Sin embargo, fuera de África, Plasmodium vivax es la especie más extendida, con altos índices de prevalencia en América y Asia $(3,4)$. Actualmente, gracias al avance en la obtención de los genomas, transcriptomas y proteomas, se ha logrado elucidar un número importante de antígenos de estas especies parasitarias que podrían estar involucrados en el proceso de invasión a células diana en el hospedero humano (5-9).

Estudios funcionales de estos antígenos han sido pieza clave para la postulación de candidatos vacunales (10-22). Las vacunas de la fase eritrocítica están destinadas a reducir la multiplicación y crecimiento del parásito, además de proteger contra los síntomas clínicos y el desarrollo de enfermedad
(23). Algunos estudios evidencian que el uso de antígenos recombinantes del parásito induciría la producción de anticuerpos capaces de bloquear la invasión de Plasmodium a eritrocitos y demostraría una eficacia protectiva en diferentes modelos animales (24-30).

De esta manera, un número importante de investigaciones se han enfocado en el análisis funcional de potenciales candidatos a vacuna de $P$. falciparum y $P$. vivax, mediante la expresión de proteínas recombinantes (PR) en sistemas heterólogos procariotas, eucariotas, y libres de células (31-34), para obtener moléculas proteicas con características semejantes a las nativas (proteínas funcionales con estructura tridimensional por los puentes disulfuro y las interacciones no covalentes) (31).

Algunos criterios para escoger un método adecuado se basan en las propiedades biológicas y químicas de los antígenos, como la secuencia codificante, para determinar regiones repetitivas y uso de codones preferenciales; al igual que la secuencia de aminoácidos, para identificar peso molecular, tipo de modificaciones postraduccionales y destino (extracelular o intracelular) (35-38). Otras consideraciones adicionales se deben tener en cuenta, tal como las condiciones de operación que requiera cada sistema, la escala de producción según el objeto de estudio y el costo en la producción, que incluye la extracción y purificación de proteínas $(39,40)$. 
Esta revisión describe las características más importantes de los sistemas de expresión más utilizados para la obtención de PR en células procariotas (cepas de Escherichia coli), células eucariotas (células de mamífero) y sistemas libres de células, empleados en estudios funcionales de antígenos de $P$. falciparum y $P$. vivax. De igual forma, se analizan los aspectos metodológicos para lograr obtener moléculas plegadas de forma nativa y funcionalmente activas.

\section{METODOLOGíA}

Se llevó a cabo una revisión descriptiva sobre sistemas de expresión de proteínas recombinantes procariotas (células bacterianas), eucariotas (células de mamífero) y sistemas libres de células utilizados en estudios funcionales de antígenos de especies $P$. falciparum y $P$. vivax, publicados en revistas indexadas en las bases de datos Embase (https://www.elsevier.com/solutions/embase-biomedical-research), Medline (https://www.ebsco. $\mathrm{com} /$ products/research-databases/medline-complete), Pubmed (https://pubmed.ncbi.nlm.nih.gov/) y Science Direct (https://www.sciencedirect.com/).

Se tuvieron en cuenta criterios de inclusión, como expresión de antígenos involucrados en la invasión a eritrocitos del hospedero humano (fase asexual del parásito) y la descripción completa del sistema de expresión empleado; asimismo, se seleccionaron artículos disponibles publicados en la ventana de tiempo establecida entre los años 2010 y 2020, escritos en idioma inglés. Se utilizaron los descriptores en Ciencias de la Salud (DeCS) y Medical Subject Headings (MeSH) para la elección de las palabras clave: antígenos, malaria, Plasmodium falciparum, Plasmodium vivax, proteínas y recombinantes.

De este modo, se analizaron 70 artículos originales, de los cuales se seleccionaron 58 , que explican en la metodología el uso de los sistemas de expresión seleccionados. Se excluyeron 12, por utilizar otros sistemas de expresión heterólogos o por no detallar el método de obtención de la recombinante. Con los artículos escogidos, se elaboró una matriz bibliográfica en Excel, organizada por título, base de datos, autor, palabras clave, resumen del artículo, fecha de publicación, particularidades del antígeno objeto de estudio y tipo de sistema de expresión utilizado. A partir de esa matriz, se generó una tabla con las características y los aspectos metodológicos tenidos en cuenta para la expresión de antígenos de Plasmodium spp.

\section{RESULTADOS}

\section{Análisis funcional de antígenos de Plas- modium spp.}

El análisis de proteínas clave en el proceso de invasión de Plasmodium spp. a las células diana se enfoca principalmente en tres pasos 
fundamentales: el análisis bioinformático, la caracterización básica y la caracterización funcional. En el primero se utilizan herramientas computacionales de alto rendimiento para analizar las proteínas del parásito a partir del genoma, transcriptoma y proteoma, con el fin de conocer los posibles candidatos a vacuna en función de su expresión génica y proteica (5-7).

Así mismo, la caracterización básica se realiza mediante un análisis bioinformático (in silico) para identificar las regiones altamente conservadas que podrían estar bajo restricción funcional. Esta observación permite eliminar las moléculas o fragmentos irrelevantes de las proteínas de $P$. falciparum y $P$. vivax que se van a estudiar (41). Posteriormente, se validan los datos obtenidos a partir de los patrones de expresión y de selección de los antígenos de estudio, investigando la capacidad de dichos fragmentos para unirse específicamente a las células blanco de infección (eritrocitos).

En esta última fase, se requieren análisis in vitro e in vivo para identificar las regiones mínimas del antígeno involucradas en la interacción proteína-célula (41). La producción de proteínas recombinantes es necesaria para desarrollar ensayos funcionales que incluyen la unión a las células diana, inhibición de la unión, inhibición de la invasión, producción de anticuerpos policlonales y experimentación en modelos animales $(31,42-44)$.
De esta forma, la producción de PR es un aspecto determinante en el análisis de candidatos a vacuna contra la malaria. A efectos de obtener estas proteínas, el gen que codifica la proteína de interés se introduce en un plásmido para facilitar su manejo y, a partir de ahí, se transfiere al sistema de expresión donde se producirá la proteína (40). Este tipo de vectores se caracteriza por transportar el material génico y protegerlo de su degradación antes de alcanzar la célula diana; además, cuenta con regiones específicas que permiten su replicación en el hospedero, así como secuencias que permiten la transcripción, la traducción y la identificación del gen clonado $(39,45)$.

Actualmente, existen sistemas de expresión recombinante basados en microrganismos y líneas celulares establecidas para su manipulación en el laboratorio. El sistema basado en bacterias (E. coli) es uno de los más utilizados, por su fácil manejo y por su elevado rendimiento (46-48). Sin embargo, la producción de proteínas del parásito Plasmodium requiere, muchas veces, modificaciones postraduccionales que este sistema no genera. Por lo tanto, en los últimos años se ha buscado producir proteínas con una estructura y función igual a la nativa, modificando genéticamente líneas de células procariotas o utilizando sistemas de expresión de eucariotas más complejos y costosos (tabla 1), como levaduras, células de insecto, células de mamífero o plantas, y recientemente sistemas libre de células (in vitro) $(38,48)$. 
Tabla 1. Características de los sistemas de expresión procariota, eucariota y libres de células

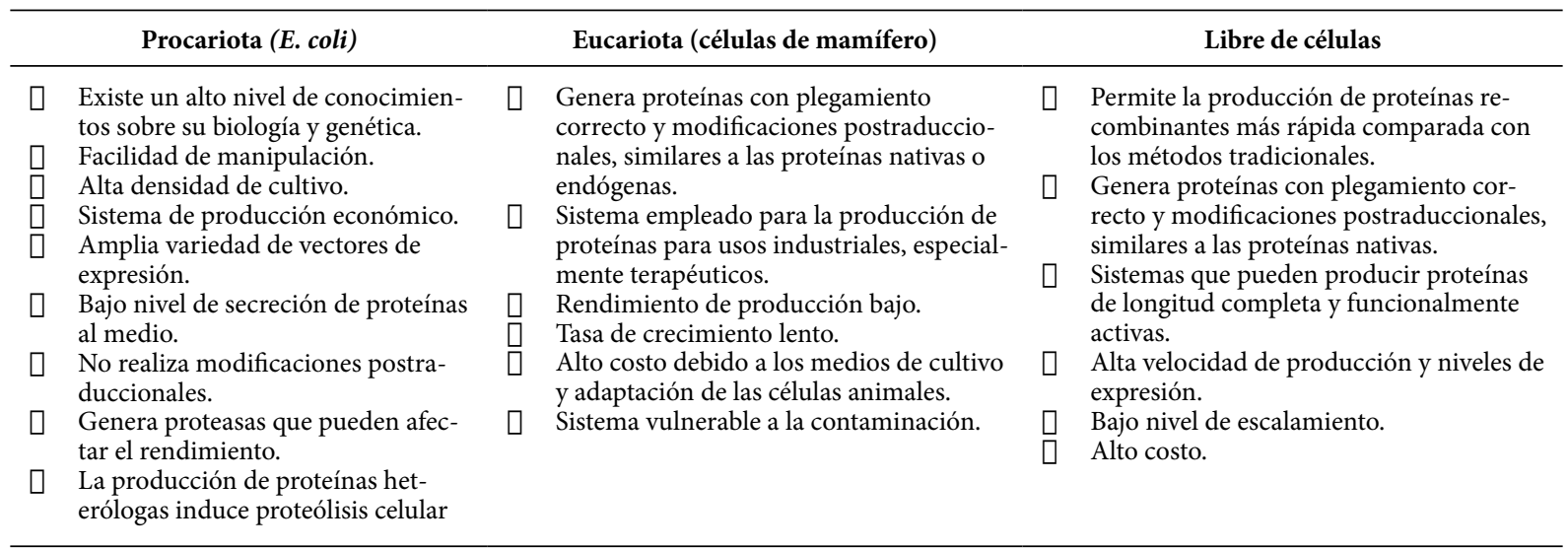

En las siguientes secciones se describen algunos de los sistemas de expresión de PR más utilizados para el estudio de potenciales candidatos a vacuna contra el estadio intraeritrocítico de parásitos de la malaria humana.

\section{Expresión de proteínas recombinantes en el sistema procariota: $E$. coli}

Uno de los métodos de producción de PR más utilizados en la obtención de antígenos de Plasmodium spp. es el sistema procariota, mediante la bacteria E. coli. Esta plataforma ofrece una serie de ventajas importantes, sobre todo relacionadas con el conocimiento del genoma, la fisiología y el metabolismo de esta bacteria, lo que facilita la manipulación genética y la generación de diferentes cepas para la producción de recombinantes $(47,48)$. Sin embargo, este método presenta limitaciones, como la falta de modificaciones postraduccionales y un inadecuado plegamiento en las proteínas generadas, lo que lleva a la implementación de pasos adicionales al proceso de obtención de PR y que hacen que el sistema se vuelva complejo y demorado.

Particularmente, los genes de $P$. falciparum, al presentar un alto contenido adenina/timina $(\mathrm{A} / \mathrm{T})$ versus citocina/guanina $(\mathrm{C} / \mathrm{G})$, así como un número importante de repeticiones de lisina y arginina en su genoma, producen la terminación temprana del proceso de traducción de ARNm (49). Por lo tanto, la mayoría de las PR derivadas de este parásito se expresan solo como formas truncadas o aparecen como cuerpos de inclusión insolubles dentro de las células bacterianas (11,50-54). 
Es interesante cómo en los últimos años el rendimiento bacteriano para la producción de PR ha mejorado notablemente, gracias al desarrollo de nuevas metodologías genéticas. Estas incluyen la modificación o eliminación de genes que codifican para la producción de proteasas, la implementación de promotores de bacteriófagos T7, la polimerasa para lograr concentraciones altas de expresión y el diseño de cepas para controlar la expresión basal (BL21 (DE3) pLysS) o la correcta formación de enlaces disulfuro (Origami, Shuffle o CyDisCo) $(46,47)$.

Así, el uso de este sistema con cepas modificadas ha permitido identificar asociaciones entre las proteínas de superficie del merozoito de $P$. falciparum (MSP1, MSP3, MSP6 y MSP7) y las proteínas asociadas con roptrias (RAP2) y con el antígeno de repeticiones de serina ubicado en los micronemas (SERA5), implicadas en el contacto inicial del parásito al eritrocito, y que han resultado ser los futuros componentes de las vacunas antimaláricas (55). De esta forma, la obtención del antígeno de unión a eritrocitos EBA-175 de forma recombinante ha permitido profundizar en la interacción de alta afinidad con el receptor glicoforina $A$ en la superficie de eritrocitos y determinar las diferentes rutas de invasión en las que participa este parásito (56).

En el caso de expresión de proteínas $P$. vivax en E.coli, especialmente las proteínas de unión a reticulocitos 1a y 2c ( $R B P 1 a$ y $R B P 2 c)$, ha favorecido la identificación de la región $\mathrm{N}$-terminal, como el fragmento de unión a células diana, y se han establecido estas regiones como objetivos de anticuerpos adquiridos de forma natural (57). Así mismo, este sistema permitió identificar las regiones de unión del complejo RON2 (RON2, 4 y 5) con el antígeno apical de merozoito-AMA1 durante la formación de la unión estrecha entre el parásito y los eritrocitos $(14,58)$. Ello ha evidenciado puntos clave de intervención que bloqueen este tipo de interacciones.

\section{Expresión de proteínas recombinantes en células de mamífero}

El uso de células de mamífero para la expresión de proteínas recombinantes se basa principalmente en la inserción de genes (transfección) de forma transitoria o estable en líneas adaptadas para este tipo de procedimientos, como células de riñón embrionario humano (HEK293) o de ovario de hámster chino $(\mathrm{CHO})(38,59,60)$. La expresión transitoria consiste en la expresión de genes sin una previa integración al ADN cromosómico de la célula hospedera. En comparación, la expresión estable demanda que un porcentaje de las células incorporen el ADN foráneo en un locus cromosómico transcripcionalmente activo y exprese el gen de forma permanente de generación en generación (61). 
Las proteínas intracelulares o transmembranales de Plasmodium se expresan principalmente de forma transitoria, utilizando vectores que contienen fuertes promotores o potenciadores, como el citomegalovirus humano y que presentan orígenes de replicación del virus de simio 40 (SV40), lo que asegura altos niveles de regulación y expresión génica (38). Actualmente, existe una amplia disponibilidad de técnicas físicas (electroporación, biobalística y microinyección), químicas (liposomas, fosfato de calcio y polímeros catiónico) y biológicas (adenovirus, retrovirus y lentivirus) para la introducción de ADN en estas células, así como medios de cultivo eficientes para las condiciones de cultivo celular (60).

El sistema de expresión recombinante con células de mamífero se ha utilizado en la producción de genotecas (ectodominios completos de proteínas) de $P$. falciparum (42 y 62 proteínas) y $P$. vivax (37 proteínas), localizadas en la superficie de merozoito o en organelos secretores invasivos $(31,62,63)$. Las proteínas generadas se caracterizaron por mantener conservada su estructura nativa; por lo tanto, se utilizaron posteriormente para estudiar las interacciones de tipo proteína-proteína principalmente entre las proteínas de superficie de membrana PfMSP7-PfMSP1, PVMSP7-PVMSP3 y P12-P41 en ambas especies parasitarias $(31,62-64)$.
Con este sistema se identificaron potenciales candidatos a vacuna, evaluando la eficacia protectora de anticuerpos contra múltiples combinaciones de antígenos de $P$. vivax (65) y regiones clave de unión del parásito a las células diana. Además, se identificó la interacción entre una proteína de P. falciparum homóloga a retoculocitos-5 ( $\mathrm{RH}-5)$ y su receptor de la superficie celular de los eritrocitos Basigina (66), que resulta ser clave en el proceso de invasión a eritrocitos $(67,68)$ y ha permitido el desarrollo de la vacuna en la etapa eritrocítica de PfRH-5(23), PfEBA (69), PfCyRPA $(20,70)$

\section{Expresión de proteínas recombinantes en sistemas libres de células}

En los últimos años, la síntesis de proteínas en sistemas libres de células (SLC) ha sido un método atractivo para la producción, de alto rendimiento, de un número importante de moléculas de Plasmodium spp. $(71,72)$. Usando maquinaria de traducción utilizada por células, se genera in vitro una reacción que programa el material genético para la expresión rápida de proteínas en pocas horas $(73,74)$.

Los principales componentes de la reacción de síntesis de proteínas en SLC se organizan en cuatro grupos: 1) la secuencia de ADN que codifica para la proteína objetivo, 2) un lisado celular (eucariota o procariota) que contenga la maquinaria 
para la transcripción y la traducción incluidos los factores de traducción y los sustratos, 3) los nucleótidos y aminoácidos y 4) los componentes necesarios para la generación de energía (35). Igualmente, se requiere la adición de diferentes compuestos químicos para crear condiciones oxidativas adecuadas, que permitan la formación de enlaces disulfuro para el correcto plegamiento de proteínas (71).

Según las características de las proteínas de estudio, el SLC dispone de una importante variedad de lisados celulares de organismos como arqueas, procariontes, hongos, plantas, insectos y mamíferos (75). Sin embargo, los extractos celulares más empleados para la obtención de proteínas a partir de los parásitos de la malaria son principalmente el de germen de trigo y el de reticulocitos de conejo, porque proporcionan un mejor rendimiento de moléculas con estructuras complejas, así como generan alta solubilidad y correcto plegamiento de muchos tipos de proteínas de alto peso molecular $(37,76,77)$.

Paradójicamente, aunque la síntesis de proteínas sin células se desarrolló hace más de 60 años, es de cierta forma reciente su uso en estudios de malaria, comparado con otros sistemas. Los análisis de inmunorreactividad y patrones de transcripción de 89 proteínas de $P$. vivax mediante microarrays identificaron un total de 18 antígenos que muestran altas respuestas de anticuerpos lgG en análisis de sueros de pacientes infectados con P. vivax (76). De igual modo, Leu et al., en 2014, identificaron 44 antígenos candidatos inmunodominantes del genoma de $P$. vivax, al producir 152 proteínas con el sistema libre de células y utilizar matrices de proteínas con sueros de pacientes con malaria, y así concluyeron que la mayoría de las proteínas presenta un perfil de inmunorreactividad alto, mayor al $80 \%$ (77). Estos resultados muestran una ventaja importante del sistema, al permitir la síntesis y estudio de varias proteínas de forma simultánea $(78,79)$. Sin embargo, estas ventajas en velocidad y flexibilidad se ven, en la mayoría de los casos, opacadas por la escasa cantidad de proteína que se produce (microgramos por mililitro).

Recientes estudios sobre nuevos candidatos a vacunas contra la malaria a partir de $P$. falciparum revelaron la producción de 1827 proteínas recombinantes utilizando SLC, lo que representa la expresión del $30 \%$ del genoma completo de esta especie (72). Mediante la técnica AlphaScreen se detectaron las interacciones proteicas entre sueros de personas expuestas a malaria (habitantes de zonas endémicas) y 128 proteínas del parásito, que evidenciaron una importante asociación de 53 proteínas que presentan peptidoseñal, dominio transmembranal y localización en la superficie del parásito, con una respuesta inmune protectora del hospedero (72). Estos resultados concuerdan con estudios previos de 
proteínas involucradas en la invasión de eritrocitos como PfRON4, PfRON2 y PfCLAG3.1 (71,80-83) y seleccionados para una evaluación adicional como candidatos a vacunas como PfRON12 (84), PV50 (85) y PVRALP1 (86).

Finalmente, la revisión presentada evidencia que existen aún muchos retos y desafíos en la producción de proteínas recombinantes para el análisis funcional de antígenos de $P$. falciparum y $P$. vivax. En el caso de los sistemas bacterianos, se espera lograr exitosamente tanto la producción de proteínas similares a las nativas, con las modificaciones postraduccionales, como la obtención de moléculas proteicas de elevado peso molecular. Por otra parte, las perspectivas en los sistemas eucariotas están dirigidas a incrementar los niveles de expresión y disminución de costos; $y$, así mismo, en los sistemas libres de células, se proyecta aumentar el nivel de escalamiento y mejoramiento de los procesos de purificación de forma automatizada.

En todos los casos, es necesario seguir estableciendo las variables que pueden influir significativamente en la eficiencia y rendimiento de moléculas recombinantes, incluyendo, por ejemplo, el perfeccionamiento genético de las cepas, los vectores de expresión, los métodos de cultivo y los métodos de purificación de estas proteínas. Además, es importante destacar que un mejor conocimiento de los mecanismos moleculares empleados por los diferentes tipos de células para la producción, contribuirán, sin duda, a incrementar los rendimientos y abrirán nuevas opciones de procesamiento de proteínas recombinantes de Plasmodium spp.

\section{CONCLUSIONES}

Con el avance en la obtención de los genomas, transcriptomas y proteomas de especies de Plasmodium se han identificado antígenos que podrían estar involucrados en el proceso de invasión a las células hospederas humanas. Así mismo, con los desarrollos recientes en la producción sintética de genes y estrategias de clonación hay una producción rápida de proteínas recombinantes en diferentes sistemas, lo que reduce los tiempos requeridos para los ensayos funcionales que permiten identificar el rol de dichas moléculas proteicas durante la invasión del parásito a las células diana.

Los mecanismos postraduccionales y el plegamiento adecuado de las proteínas del parásito de la malaria humana son aspectos críticos para tener en cuenta durante la selección del sistema de expresión. Los métodos de producción de proteínas recombinantes basados en células procariotas de $E$. coli, células eucariotas de mamífero y sistemas libres de células han sido claves para la caracterización de un número importante de antígenos candidatos a vacuna en $P$. falciparum y $P$. 
vivax. Estos avances contribuyen al desarrollo de medidas profilácticas o terapéuticas que ayudan a mitigar la enfermedad de la malaria, un problema drástico de salud pública en todo el mundo.

\section{AGRADECIMIENTOS}

Los autores agradecen a la Universidad de Boyacá, por su apoyo en la elaboración de este estudio.

\section{LIMITACIONES}

No hubo limitaciones.

\section{CONFLICTO DE INTERESES}

Los autores declaran no tener conflicto de intereses.

\section{FINANCIACIÓN}

Los autores declaran la financiación por parte de la Universidad de Boyacá de los medios necesarios para llevar a cabo este artículo de revisión.

\section{REFERENCIAS}

1. World Health Organization. Word malaria report 2019. Washington: WHO; 2019.

2. Cowman AF, Healer J, Marapana D, MarshK. Malaria: biology and disease. Cell. 2016;167(3):610-24. https://doi.org/10.1016/j.cell.2016.07.055
3. Gething PW, Elyazar IR, Moyes $C L$, Smith $D L$, Battle KE, Guerra $C A$, et al. A long neglected world malaria map: Plasmodium vivax endemicity in 2010. PLoS Negl Trop Dis. 2012;6(9):e1814. https://doi.org/10.1371/ journal.pntd.0001814

4. Price RN, Tjitra E, Guerra CA, Yeung S, White NJ, Anstey NM. Vivax malaria: neglected and not benign. Am J Trop Med Hyg. 2007;77(6 Suppl):79-87. https://doi.org/10.4269/ ajtmh.2007.77.79

5. Venkatesh A, Patel SK, Ray S, Shastri J, Chatterjee G, Kochar SK, et al. Proteomics of Plasmodium vivax malaria: new insights, progress and potential. Expert Rev Proteom. 2016;13(8):771-82. https://doi.org/10.1080/1 4789450.2016.1210515

6. Moreno-Pérez DA, Degano R, Ibarrola N, Muro A, Patarroyo MA. Determining the Plasmodium vivax VCG-1 strain blood stage proteome. J Proteomics. 2015;113:268-80. https://doi. org/10.1016/j.jprot.2014.10.003

7. Acharya P, Pallavi R, Chandran S, Chakravarti $H$, Middha S, Acharya J, et al. A glimpse into the clinical proteome of human malaria parasites Plasmodium falciparum and Plasmodium vivax. Proteom Clin Appl. 2009;3(11):1314-25. https://doi.org/10.1002/prca.200900090 
8. Boucher MJ, Ghosh S, Zhang L, Lal A, Jang $\mathrm{SW}$, Ju $A$, et al. Integrative proteomics and bioinformatic prediction enable a highconfidence apicoplast proteome in malaria parasites. PLoS Biology. 2018;16(9):e2005895. https://doi.org/10.1371/journal.pbio.2005895

9. Bunnik EM, Batugedara G, Saraf A, Prudhomme J, Florens L, Le Roch KG. The mRNA-bound proteome of the human malaria parasite Plasmodium falciparum. Genome Biol. 2016;17(1):147. https://doi.org/10.1186/ s13059-016-1014-0

10. Alam MS, Choudhary V, Zeeshan M, Tyagi RK, Rathore S, Sharma YD. Interaction of Plasmodium vivax Tryptophan-rich Antigen PvTRAg38 with band 3 on human erythrocyte surface facilitates parasite growth. J Biol Chem. 2015;290(33):20257-72. https://doi. org/10.1074/jbc.M115.644906

11. Arevalo-Pinzon G, Curtidor H, Patino LC, Patarroyo MA. PvRON2, a new Plasmodium vivax rhoptry neck antigen. Malaria J. 2011;10:60. https://doi.org/10.1186/14752875-10-60

12. Bartholdson SJ, Bustamante LY, Crosnier C, Johnson S, Lea S, Rayner JC, et al. Semaphorin7A is an erythrocyte receptor for $P$. falciparum merozoite-specific TRAP homolog, MTRAP.
PLoS Pathogens. 2012;8(11):e1003031. https://doi.org/10.1371/journal.ppat.1003031

13. Batchelor JD, Malpede BM, Omattage NS, DeKoster GT, Henzler-Wildman KA, Tolia NH. Red blood cell invasion by Plasmodium vivax: structural basis for DBP engagement of DARC. PLoS pathogens. 2014;10(1):e1003869. https://doi.org/10.1371/journal.ppat.1003869

14. Bermudez $M$, Arevalo-Pinzon $G$, Rubio $\mathrm{L}$, Chaloin O, Muller S, Curtidor $\mathrm{H}$, et al. Receptor-ligand and parasite protein-protein interactions in Plasmodium vivax: Analysing rhoptry neck proteins 2 and 4. Cellular microbiology. 2018;20(7):e12835. https://doi. org/10.1111/cmi.12835

15. Chen Q, Pettersson F, Vogt AM, Schmidt B, Ahuja $S$, Liljestrom $P$, et al. Immunization with PfEMP1-DBL1alpha generates antibodies that disrupt rosettes and protect against the sequestration of Plasmodium falciparuminfected erythrocytes. Vaccine. 2004;22(2122):2701-12. https://doi.org/10.1016/j. vaccine.2004.02.015

16. Cheng Y, Lu F, Tsuboi T, Han ET. Characterization of a novel merozoite surface protein of Plasmodium vivax, Pv41. Acta Tropica. 2013;126(3):222-8. https://doi.org/10.1016/j. actatropica.2013.03.002 
17. Douglas $A D$, Williams $A R$, Knuepfer $E$, Illingworth JJ, Furze JM, Crosnier C, et al. Neutralization of Plasmodium falciparum merozoites by antibodies against PfRH5. J Immunol. 2014;192(1):245-58. https://doi. org/10.4049/jimmunol.1302045

18. Duraisingh MT, Maier AG, Triglia $T$, Cowman AF. Erythrocyte-binding antigen 175 mediates invasion in Plasmodium falciparum utilizing sialic acid-dependent and -independent pathways. Proc Natl Acad Sci U S A. 2003;100(8):4796-801. https://doi. org/10.1073/pnas.0730883100

19. Dundas K, Shears MJ, Sun Y, Hopp CS, Crosnier $\mathrm{C}$, Metcalf $\mathrm{T}$, et al. Alpha-v-containing integrins are host receptors for the Plasmodium falciparum sporozoite surface protein, TRAP. Proc Natl Acad Sci U S A. 2018;115(17):447782. https://doi.org/10.1073/pnas.1719660115

20. Favuzza P, Guffart E, Tamborrini M, Scherer $B$, Dreyer AM, Rufer AC, et al. Structure of the malaria vaccine candidate antigen CyRPA and its complex with a parasite invasion inhibitory antibody. eLife. 2017;6. https://doi. org/10.7554/eLife.20383

21. Khattab A, Bonow I, Schreiber N, Petter M, Schmetz C, Klinkert MQ. Plasmodium falciparum variant STEVOR antigens are expressed in merozoites and possibly associated with erythrocyte invasion. Malaria J. 2008;7:137. https://doi.org/10.1186/14752875-7-137

22. Rathore S, Dass S, Kandari D, Kaur I, Gupta M, Sharma YD. Basigin interacts with Plasmodium vivax Tryptophan-rich Antigen PvTRAg38 as a second erythrocyte receptor to promote parasite growth. J Biol Chem. 2017;292(2):462-76. https://doi.org/10.1074/ jbc.M116.744367

23. Salamanca $D R$, Gómez $M$, Camargo $A$, Cuy-Chaparro L, Molina-Franky J, Reyes C, et al. Plasmodium falciparum blood stage antimalarial vaccines: an analysis of ongoing clinical trials and new perspectives related to synthetic vaccines. Front Microbiol. 2019;10:2712. https://doi.org/10.3389/ fmicb.2019.02712

24. Alanine DGW, Quinkert D, Kumarasingha $R$, Mehmood S, Donnellan FR, Minkah NK, et al. Human antibodies that slow erythrocyte invasion potentiate malaria-neutralizing antibodies. Cell. 2019;178(1):216-28 e21. https://doi.org/10.1016/j.cell.2019.05.025

25. Chen L, Xu Y, Wong W, Thompson JK, Healer J, Goddard-Borger ED, et al. Structural basis for inhibition of erythrocyte invasion by 
antibodies to Plasmodium falciparum protein CyRPA. eLife. 2017;6. https://doi.org/10.7554/ elife.21347

26. Chootong $P$, Ntumngia $F B$, VanBuskirk KM, Xainli J, Cole-Tobian JL, Campbell CO, et al. Mapping epitopes of the Plasmodium vivax Duffy binding protein with naturally acquired inhibitory antibodies. Infect Immun. 2010;78(3):1089-95. https://doi.org/10.1128/ IAI.01036-09

27. Gao X, Yeo KP, Aw SS, Kuss C, lyer JK, Genesan $S$, et al. Antibodies targeting the PfRH1 binding domain inhibit invasion of Plasmodium falciparum merozoites. PLoS Pathogens. 2008;4(7):e1000104. https://doi. org/10.1371/journal.ppat.1000104

28. Healer J, Thompson JK, Riglar DT, Wilson DW, Chiu YH, Miura K, et al. Vaccination with conserved regions of erythrocyte-binding antigens induces neutralizing antibodies against multiple strains of Plasmodium falciparum. PLoS One. 2013;8(9):e72504. https://doi.org/10.1371/journal.pone.0072504

29. Nicolete VC, Frischmann S, Barbosa S, King $\mathrm{CL}$, Ferreira MU. Naturally acquired bindinginhibitory antibodies to Plasmodium vivax duffy binding protein and clinical immunity to malaria in rural Amazonians. J Infect
Dis. 2016;214(10):1539-46. https://doi. org/10.1093/infdis/jiw407

30. Zhou AE, Berry AA, Bailey JA, Pike A, Dara A, Agrawal $S$, et al. Antibodies to peptides in semiconserved domains of RIFINs and STEVORs correlate with malaria exposure. mSphere. 2019;4(2). https://doi.org/10.1128/ mSphere.00097-19

31. Hostetler JB, Sharma S, Bartholdson SJ, Wright GJ, Fairhurst RM, Rayner JC. A Library of Plasmodium vivax recombinant merozoite proteins reveals new vaccine candidates and protein-protein interactions. PLoS Negl Trop Dis. 2015;9(12):e0004264. https://doi. org/10.1371/journal.pntd.0004264

32. Draper SJ, Sack BK, King CR, Nielsen CM, Rayner JC, Higgins MK, et al. Malaria vaccines: recent advances and new horizons. Cell Host Microbe. 2018;24(1):43-56. https://doi. org/10.1016/j.chom.2018.06.008

33. Singh $K$, Mukherjee $P$, Shakri AR, Singh $A$, Pandey $G$, Bakshi $M$, et al. Malaria vaccine candidate based on Duffy-binding protein elicits strain transcending functional antibodies in a Phase I trial. NPJ Vaccines. 2018;3:48. https://doi.org/10.1038/s41541018-0083-3 
34. Zheng J, Pan H, Gu Y, Zuo X, Ran N, Yuan $Y$, et al. Prospects for malaria vaccines: pre-erythrocytic stages, blood stages, and transmission-blocking stages. Biomed Res Int. 2019;2019:9751471. https://doi. org/10.1155/2019/9751471

35. Yadavalli R, Ledger C, Sam-Yellowe TY. In vitro human cell-free expression system for synthesis of malaria proteins. Parasitol Res. 2012;111(6):2461-5. https://doi.org/10.1007/ s00436-012-3014-7

36. Srivastava A, Durocher $Y$, Gamain $B$. Expressing full-length functional PfEMP1 proteins in the HEK293 expression system. Methods Mol Biol. 2013;923:307-19. https:// doi.org/10.1007/978-1-62703-026-7_22

37. Zemella A, Thoring L, Hoffmeister C, Kubick S. Cell-free protein synthesis: pros and cons of prokaryotic and eukaryotic systems. Chembiochem. 2015;16(17):2420-31. https:// doi.org/10.1002/cbic. 201500340

38. Hacker DL, Balasubramanian S. Recombinant protein production from stable mammalian cell lines and pools. Curr Opin Struct Biol. 2016;38:12936. https://doi.org/10.1016/j.sbi.2016.06.005

39. Wingfield PT. Overview of the purification of recombinant proteins. Curr Protoc Protein
Sci. 2015;80:6.1.1-6.1.35. https://doi. org/10.1002/0471140864.ps0601s80

40. Ferrer-Miralles N, Saccardo $\mathrm{P}$, Corchero JL, Xu Z, Garcia-FruitosE. General introduction: recombinant protein production and purification of insoluble proteins. Methods Mol Biol. 2015;1258:1-24. https://doi.org/10.1007/978-1-4939-2205-5_1

41. Patarroyo MA, Arevalo-Pinzon G, MorenoPerez DA. From a basic to a functional approach for developing a blood stage vaccine against Plasmodium vivax. Expert Rev Vaccines. 2020;19(2):195-207. https://doi.org/ 10.1080/14760584.2020.1733421

42. Sirima SB, Durier C, Kara L, Houard S, Gansane A, Loulergue $P$, et al. Safety and immunogenicity of a recombinant Plasmodium falciparum AMA1-DiCo malaria vaccine adjuvanted with GLA-SE or Alhydrogel(R) in European and African adults: a phase $1 \mathrm{a} / 1 \mathrm{~b}$, randomized, double-blind multi-centre trial. Vaccine. 2017;35(45):6218-27. https://doi. org/10.1016/j.vaccine.2017.09.027

43. Gaur D, Singh S, Singh S, Jiang L, Diouf A, Miller LH. Recombinant Plasmodium falciparum reticulocyte homology protein 4 binds to erythrocytes and blocks invasion. Proc Natl Acad Sci U S A. 2007;104(45):17789-94. https://doi.org/10.1073/pnas.0708772104 
44. Rosa DS, Iwai LK, Tzelepis F, Bargieri DY, Medeiros MA, Soares IS, et al. Immunogenicity of a recombinant protein containing the Plasmodium vivax vaccine candidate MSP1(19) and two human CD4+ T-cell epitopes administered to non-human primates (Callithrix jacchus jacchus). Microbes Infect. 2006;8(8):2130-7. https://doi.org/10.1016/j. micinf.2006.03.012

45. Gileadi O. Recombinant Protein Expression in E. coli: a historical perspective. Methods Mol Biol. 2017;1586:3-10. https://doi. org/10.1007/978-1-4939-6887-9_1

46. Gopal GJ, Kumar A. Strategies for the production of recombinant protein in Escherichia coli. Protein J. 2013;32(6):419-25. https://doi.org/10.1007/s10930-013-9502-5

47. Rosano GL, Morales ES, Ceccarelli EA. New tools for recombinant protein production in Escherichia coli: A 5-year update. Protein Sci. 2019;28(8):1412-22. https://doi.org/10.1002/ pro.3668

48. Hayat SMG, Farahani N, Golichenari B, Sahebkar A. Recombinant Protein Expression in Escherichia coli (E.coli): What We Need to Know. Curr Pharm Des. 2018;24(6):718-25. https://doi.org/10.2174/13816128246661801 31121940
49. Flick K, Ahuja S, Chene A, Bejarano MT, Chen Q. Optimized expression of Plasmodium falciparum erythrocyte membrane protein 1 domains in Escherichia coli. Malaria J. 2004;3:50. https://doi.org/10.1186/14752875-3-50

50. Reddy KS, Amlabu E, Pandey AK, Mitra P, Chauhan VS, Gaur D. Multiprotein complex between the GPI-anchored CyRPA with PfRH5 and PfRipr is crucial for Plasmodium falciparum erythrocyte invasion. Proc Natl Acad Sci U S A. 2015;112(4):1179-84. https:// doi.org/10.1073/pnas.1415466112

51. Ntumngia FB, Thomson-Luque R, Torres Lde M, Gunalan K, Carvalho LH, Adams JH. A novel erythrocyte binding protein of Plasmodium vivax suggests an alternate invasion pathway into duffy-positive reticulocytes. mBio. 2016;7(4). https://doi.org/10.1128/ mBio.01261-16

52. Muh F, Han JH, Nyunt MH, Lee SK, Jeon HY, Ha $\mathrm{KS}$, et al. Identification of a novel merozoite surface antigen of Plasmodium vivax, PvMSA180. Malaria J. 2017;16(1):133. https:// doi.org/10.1186/s12936-017-1760-9

53. Arévalo-Pinzón $G$, Bermúdez $M$, Curtidor $H$, Patarroyo MA. The Plasmodium vivax rhoptry neck protein 5 is expressed in the apical pole of 
Plasmodium vivax VCG-1 strain schizonts and binds to human reticulocytes. Malaria J. 2015;14:106. https://doi.org/10.1186/s12936-015-0619-1

54. Arevalo-Pinzon G, Curtidor H, Abril J, Patarroyo MA. Annotation and characterization of the Plasmodium vivax rhoptry neck protein 4 (PvRON4). Malaria J. 2013;12:356. https://doi. org/10.1186/1475-2875-12-356

55. Deshmukh A, Chourasia BK, Mehrotra S, Kana IH, Paul G, Panda A, et al. Plasmodium falciparum MSP3 exists in a complex on the merozoite surface and generates antibody response during natural infection. Infect Immun. 2018;86(8). https://doi.org/10.1128/ IAI.00067-18

56. Sisquella X, Nebl T, Thompson JK, Whitehead L, Malpede BM, Salinas ND, et al. Plasmodium falciparum ligand binding to erythrocytes induce alterations in deformability essential for invasion. elife. 2017;6. https://doi. org/10.7554/eLife.21083

57. Gupta ED, Anand G, Singh H, Chaddha K, Bharti PK, Singh $N$, et al. Naturally acquired human antibodies against reticulocyte-binding domains of Plasmodium vivax proteins, PvRBP2c and PVRBP1a, exhibit binding-inhibitory activity. J Infect Dis. 2017;215(10):1558-68. https://doi.org/10.1093/infdis/jix170
58. Arévalo-Pinzón G, Bermúdez M, Hernández D, Curtidor $\mathrm{H}$, Patarroyo MA. Plasmodium vivax ligand-receptor interaction: PvAMA-1 domain I contains the minimal regions for specific interaction with $\mathrm{CD} 71+$ reticulocytes. Sci Rep. 2017;7(1):9616. https://doi.org/10.1038/ s41598-017-10025-6

59. Chen S, Gray D, Ma J, Subramanian S. Production of recombinant proteins in mammalian cells. Curr Protoc Protein Sci. 2001; Chapter 5:Unit5 10. https://doi. org/10.1002/0471140864.ps0510s12

60. Bandaranayake AD, Almo SC. Recent advances in mammalian protein production. FEBS letters. 2014;588(2):253-60. https://doi. org/10.1016/j.febslet.2013.11.035

61. O'Flaherty R, Bergin A, Flampouri E, Mota LM, Obaidi I, Quigley A, et al. Mammalian cell culture for production of recombinant proteins: A review of the critical steps in their biomanufacturing. Biotechnol Adv. 2020:107552. https://doi.org/10.1016/j. biotechadv.2020.107552

62. Crosnier C, Wanaguru M, McDade B, Osier FH, Marsh K, Rayner JC, et al. A library of functional recombinant cell-surface and secreted P. falciparum merozoite proteins. Mol 
Cell Proteomics. 2013;12(12):3976-86. https:// doi.org/10.1074/mcp.0113.028357

63. Zenonos ZA, Rayner JC, Wright GJ. Towards a comprehensive Plasmodium falciparum merozoite cell surface and secreted recombinant protein library. Malaria J. 2014;13:93. https://doi.org/10.1186/14752875-13-93

64. Franca CT, He WQ, Gruszczyk J, Lim NT, Lin $E$, Kiniboro $B$, et al. Plasmodium vivax reticulocyte binding proteins are key targets of naturally acquired immunity in young Papua New Guinean children. PLoS Negl Trop Dis. 2016;10(9):e0005014. https://doi. org/10.1371/journal.pntd.0005014

65. Franca $C T$, White $M T$, He WQ, Hostetler $J B$, Brewster J, Frato $G$, et al. Identification of highly-protective combinations of Plasmodium vivax recombinant proteins for vaccine development. eLife. 2017;6. https:// doi.org/10.7554/eLife. 28673

66.Crosnier C, Bustamante LY, Bartholdson SJ, Bei AK, Theron M, Uchikawa M, et al. Basigin is a receptor essential for erythrocyte invasion by Plasmodium falciparum. Nature. 2011;480(7378):534-7. https://doi. org/10.1038/nature10606
67. Rodriguez M, Lustigman S, Montero E, Oksov $Y$, Lobo CA. PfRH5: a novel reticulocytebinding family homolog of plasmodium falciparum that binds to the erythrocyte, and an investigation of its receptor. PLOS One. 2008;3(10):e3300. https://doi.org/10.1371/ journal.pone. 0003300

68. Arevalo-Pinzon $G$, Curtidor $H$, Munoz $M$, Patarroyo MA, Bermudez A, Patarroyo ME. A single amino acid change in the Plasmodium falciparum RH5 (PfRH5) human RBC binding sequence modifies its structure and determines species-specific binding activity. Vaccine. 2012;30(3):637-46. https://doi.org/10.1016/j. vaccine.2011.11.012

69. Wanaguru M, Crosnier C, Johnson S, Rayner JC, Wright GJ. Biochemical analysis of the Plasmodium falciparum erythrocytebinding antigen-175 (EBA175)-glycophorin-A interaction: implications for vaccine design. J Biol Chem. 2013;288(45):32106-17. https:// doi.org/10.1074/jbc.M113.484840

70. Tamborrini M, Hauser J, Schafer A, Amacker M, Favuzza $P$, Kyungtak $K$, et al. Vaccination with virosomally formulated recombinant CyRPA elicits protective antibodies against Plasmodium falciparum parasites in preclinical in vitro and in vivo models. NPJ Vaccines. 2020;5:9. https:// doi.org/10.1038/s41541-020-0158-9 
71. Morita M, Takashima E, Ito D, Miura K, Thongkukiatkul A, Diouf A, et al. Immunoscreening of Plasmodium falciparum proteins expressed in a wheat germ cellfree system reveals a novel malaria vaccine candidate. Sci Rep. 2017;7:46086. https://doi. org/10.1038/srep46086

72. Kanoi BN, Takashima E, Morita M, White MT, Palacpac NM, Ntege EH, et al. Antibody profiles to wheat germ cell-free system synthesized Plasmodium falciparum proteins correlate with protection from symptomatic malaria in Uganda. Vaccine. 2017;35(6):873-81. https:// doi.org/10.1016/j.vaccine.2017.01.001

73. Takeda M, Kainosho M. Cell-free protein production for NMR studies. Methods Mol Biol. 2012;831:71-84. https://doi.org/10.1007/9781-61779-480-3_5

74. Tsuboi T, Takeo S, Iriko H, Jin L, Tsuchimochi $M$, Matsuda $S$, et al. Wheat germ cell-free system-based production of malaria proteins for discovery of novel vaccine candidates. Infect Immun. 2008;76(4):1702-8. https://doi. org/10.1128/IAI.01539-07

75. Yadavalli R, Sam-Yellowe T. HeLa based cell free expressionsystemsforexpression of Plasmodium rhoptry proteins. J Vis Exp. 2015(100):e52772. https://doi.org/10.3791/52772
76. Chen JH, Jung JW, Wang Y, Ha KS, Lu F, Lim CS, et al. Immunoproteomics profiling of blood stage Plasmodium vivax infection by highthroughput screening assays. J Proteome Res. 2010;9(12):6479-89. https://doi.org/10.1021/ pr100705g

77. Lu F, Li J, Wang B, Cheng Y, Kong DH, Cui L, et al. Profiling the humoral immune responses to Plasmodium vivax infection and identification of candidate immunogenic rhoptry-associated membrane antigen (RAMA). J Proteomics. 2014;102:66-82. https://doi.org/10.1016/j. jprot.2014.02.029

78. Arévalo-Pinzón G, González-González $M$, Suárez CF, Curtidor $\mathrm{H}$, Carabias-Sánchez J, Muro $A$, et al. Self-assembling functional programmable protein array for studying protein-protein interactions in malaria parasites. Malaria J. 2018;17(1):270. https:// doi.org/10.1186/s12936-018-2414-2

79. Takeo S, Arumugam TU, Torii M, Tsuboi T. Wheat germ cell-free technology for accelerating the malaria vaccine research. Expert Opin Drug Discov. 2009;4(11):1191-9. https://doi.org/10.1517/17460440903369813

80. Curtidor H, Patino LC, Arévalo-Pinzón G, Patarroyo ME, Patarroyo MA. Identification of the Plasmodium falciparum rhoptry neck 
protein 5 (PfRON5). Gene. 2011;474(1-2):228. https://doi.org/10.1016/j.gene.2010.12.005

81. Hossain ME, Dhawan S, Mohmmed A. The cysteine-rich regions of Plasmodium falciparum RON2 bind with host erythrocyte and AMA1 during merozoite invasion. Parasitol Res. 2012;110(5):1711-21. https:// doi.org/10.1007/s00436-011-2690-z

82. Quintana MDP, Ch'ng JH, Zandian A, Imam $M$, Hultenby $K$, Theisen $M$, et al. SURGE complex of Plasmodium falciparum in the rhoptry-neck (SURFIN4.2-RON4-GLURP) contributes to merozoite invasion. PLoS One. 2018;13(8):e0201669. https://doi. org/10.1371/journal.pone.0201669

83. Gardiner DL, Spielmann T, Dixon MW, Hawthorne PL, Ortega MR, Anderson KL, et al. CLAG 9 is located in the rhoptries of Plasmodium falciparum. Parasitol Res. 2004;93(1):64-7. https://doi.org/10.1007/ s00436-004-1098-4

84. Ito D, Takashima E, Yamasaki T, Hatano $S$, Hasegawa T, Miura K, et al. Antibodies against a Plasmodium falciparum RON12 inhibit merozoite invasion into erythrocytes. Parasitol Int. 2019;68(1):87-91. https://doi. org/10.1016/j.parint.2018.10.006
85. Cheng Y, Wang B, Lu F, Ahmed MA, Han JH, Na $\mathrm{SH}$, et al. Identification and characterization of Pv50, a novel Plasmodium vivax merozoite surface protein. Parasit Vectors. 2019;12(1):176. https://doi.org/10.1186/ s13071-019-3434-7

86. Cheng $Y$, Li J, Ito D, Kong DH, Ha KS, Lu F, et al. Antigenicity and immunogenicity of PvRALP1, a novel Plasmodium vivax rhoptry neck protein. Malaria J. 2015;14:186. https:// doi.org/10.1186/s12936-015-0698-z

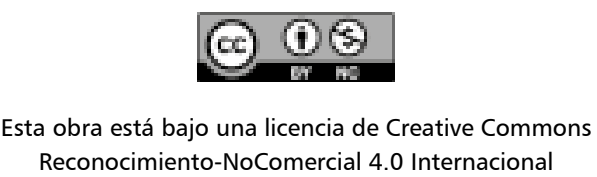

Esta obra está bajo una licencia de Creative Commons 